\title{
Discrete $(h, k)$-Dichotomy and Remarks on the Boundedness of the Projections
}

\author{
Mihai-Gabriel Babuţia, ${ }^{1}$ Monteola Ilona Kovács, ${ }^{1}$ Mărioara Lăpădat, ${ }^{1}$ and Mihail Megan ${ }^{2}$ \\ ${ }^{1}$ Department of Mathematics, Faculty of Mathematics and Computer Science, West University of Timişoara, \\ 4 Parvan Boulevard, 300223 Timișoara, Romania \\ ${ }^{2}$ Academy of Romanian Scientists, 54 Independenţei Street, 050094 Bucharest, Romania
}

Correspondence should be addressed to Mihail Megan; megan@math.uvt.ro

Received 18 November 2013; Revised 29 May 2014; Accepted 9 June 2014; Published 6 July 2014

Academic Editor: Jan Lang

Copyright (c) 2014 Mihai-Gabriel Babuţia et al. This is an open access article distributed under the Creative Commons Attribution License, which permits unrestricted use, distribution, and reproduction in any medium, provided the original work is properly cited.

The present paper treats a concept of $(h, k)$-dichotomy for linear discrete systems. Sufficient conditions for the $k$-boundedness of the projection sequences that give the dichotomy are presented and an illustrative example shows the connection between the growth of the system and the bound of the sequence of projections. Thus the growth of the system that is assumed in the theorems is essential.

\section{Introduction}

Among the asymptotic behaviors of discrete linear systems, an important role is played by the dichotomy property (see [1-12] and the references therein).

A natural generalization of both the uniform and nonuniform dichotomy is successfully modeled by the concept of $(h, k)$-dichotomy, where a significant number of papers containing many interesting results were published, from which we mention $[5,13-15]$. In recent years, several papers, also dedicated to asymptotic behavior of discrete dynamical systems, appeared and hence the generalization to $(h, k)$ behavior is present even for the trichotomy property (see $[3,8,9,15-17])$. Among the results that were obtained in this field, we concentrate our attention on the property of boundedness of the sequence of the projections that give the dichotomic behavior. For example, in [10], in the case of discrete variational systems, the uniform exponential dichotomy property of a discrete cocycle $\{\Phi(\theta, n)\}_{\theta \in \Theta, n \in \mathbb{N}}$ which has a uniform exponential growth implies the boundedness of the dichotomic sequence of projections (here, the proof being more direct, compared with those found in the literature, without using the angular distance between the dichotomy subspaces). Further results concerning the boundedness of the dichotomy sequence of the projections are also presented in [4]. In [12], such results are proved both in the case of uniformly bounded coefficients of the discrete linear system (see Remark 3.3) and through techniques of admissibility of pairs of function spaces to the associated control system of the discrete linear system (Theorem 3.3).

This is the direction in which the present paper intends to state the results, by defining the concept of $(h, k)$-dichotomy for linear discrete systems and showing that, under similar hypotheses as stated in [10], we obtain the $k$-boundedness of the sequence of projections that give the dichotomic behavior.

Bounded and exponentially bounded sequences of projections, although not explicitly stated, are widely used in the study of the exponential dichotomy, and this intrinsic property is assumed from the beginning, in the definition of the asymptotic behavior (see, e.g., [2]). Having in mind the above reasons, we present sufficient conditions of the $k$ boundedness of a sequence of projections that give the $(h, k)$ dichotomy for a given discrete linear system.

Finally, an illustrative example will show that the main assumption of $(h, k)$-growth is essential, and we present an example that, besides the property mentioned above, also shows how closely connected is the growth of the system to the boundedness of the sequence of projections. 


\section{Basic Definitions and Notations}

Let $X$ be a Banach space and $\mathscr{B}(X)$ the Banach algebra of all bounded linear operators on $X$. The norms on $X$ and on $\mathscr{B}(X)$ will be denoted by $\|\cdot\|$. The identity operator on $X$ is denoted by $I$. We also denote by $\mathbb{N}^{*}$ the set of positive integers and by $\mathbb{R}_{+}$the set of nonnegative real numbers. We also denote by

$$
\Delta=\{(m, n) \in \mathbb{N} \times \mathbb{N}: m \geq n\} .
$$

We consider the linear difference system

$$
x_{n+1}=\mathbb{A}_{n} x_{n}, \quad n \in \mathbb{N},
$$

where $\mathbb{A}: \mathbb{N} \rightarrow \mathscr{B}(X)$ is a given sequence. For $(m, n) \in \Delta$ we define

$$
A(m, n)= \begin{cases}\mathbb{A}(m-1) \cdots \mathbb{A}(n) & \text { if } m>n \\ I & \text { if } m=n .\end{cases}
$$

Definition 1. The map $A: \Delta \rightarrow \mathscr{B}(X)$ defined above is called the evolution operator associated to the system $(\mathfrak{U})$.

Remark 2. If the sequence $\left(x_{n}\right)_{n \geq 0}$ is a solution of $(\mathfrak{A})$, then

$$
x_{m}=A(m, n) x_{n}, \quad \forall(m, n) \in \Delta .
$$

Definition 3. A map $P: \mathbb{N} \rightarrow \mathscr{B}(X)$ is called a sequence of projections if

$$
P_{n} P_{n}=P_{n}, \quad \forall n \in \mathbb{N} .
$$

If $P: \mathbb{N} \rightarrow \mathscr{B}(X)$ is a sequence of projections, then the sequence $Q: \mathbb{N} \rightarrow \mathscr{B}(X)$ defined by

$$
Q_{n}=I-P_{n}, \quad \forall n \in \mathbb{N}
$$

is also a sequence of projections, called the complementary sequence of $P$.

Definition 4. A sequence $\varphi: \mathbb{N} \rightarrow[1,+\infty)$ is called a growth rate if the following conditions are satisfied:

$\left(\mathrm{gr}_{1}\right) \varphi$ is nondecreasing,

$\left(\mathrm{gr}_{2}\right) \varphi(0)=1$,

$\left(\mathrm{gr}_{3}\right) \lim _{n \rightarrow+\infty} \varphi(n)=+\infty$.

Definition 5. Let $k: \mathbb{N} \rightarrow[1,+\infty)$ be a growth rate and let $P: \mathbb{N} \rightarrow \mathscr{B}(X)$ be a sequence of projections. We say that $P$ is $k$-bounded if there exist $L>0$ and $\omega \geq 0$ such that

$$
\left\|P_{n}\right\| \leq L k(n)^{\omega} \quad \forall n \in \mathbb{N} .
$$

Remark 6. As particular cases of the $k$-boundedness, we give the following.

(i) If $k(n)=e^{n}$, that is, there exist $L>0$ and $\omega \geq 0$ such that

$$
\left\|P_{n}\right\| \leq L e^{\omega n}, \quad \forall n \in \mathbb{N},
$$

then we say that $P$ is exponentially bounded. (ii) If $k(n)=n+1$, that is, there exist $L>0$ and $\omega \geq 0$ such that

$$
\left\|P_{n}\right\| \leq L(n+1)^{\omega}, \quad \forall n \in \mathbb{N}
$$

then we say that $P$ is polynomially bounded.

(iii) If $\omega=0$, that is, there exists $L>0$ such that

$$
\left\|P_{n}\right\| \leq L, \quad \forall n \in \mathbb{N},
$$

then we say that $P$ is bounded.

Let $h, k: \mathbb{N} \rightarrow[1,+\infty)$ be two growth rates.

Definition 7. The system $(\mathfrak{U})$ is said to have a $(h, k)$-growth if there exist $M, \omega_{1}>0$ and $\omega_{2} \geq 0$ such that

$$
\|A(m, n) x\| \leq M k(n)^{\omega_{2}}\left(\frac{h(m)}{h(n)}\right)^{\omega_{1}}\|x\|
$$

for all $(m, n, x) \in \Delta \times X$.

In the particular case in which $\omega_{2}=0$, we say that the system $(\mathfrak{U})$ has a uniform $(h, k)$-growth or an $h$-growth.

Remark 8. As particular cases of (10), we have the following.

(i) If $h(n)=k(n)=e^{n}$, then we say that the system $(\mathfrak{\mathfrak { U }})$ has an exponential growth.

(ii) If $h(n)=e^{n}$, then we say that the system $(\mathfrak{\mathcal { U }})$ has a nonuniform exponential growth.

(iii) If $h(n)=e^{n}$ and $\omega_{2}=0$, then we say that the system $(\mathfrak{A})$ has a uniform exponential growth.

(iv) If $h(n)=k(n)=n+1$, then we say that the system ( $\mathfrak{X})$ has a polynomial growth.

(v) If $h(n)=n+1$, then we say that the system ( $\mathfrak{A})$ has a nonuniform polynomial growth.

(vi) If $h(n)=n+1$ and $\omega_{2}=0$, then we say that the system ( $\mathfrak{A})$ has a uniform polynomial growth.

\section{3. $(h, k)$-Dichotomy and Main Results}

Let $h, k: \mathbb{N} \rightarrow[1,+\infty)$ be two growth rates.

Definition 9. We say that the system $(\mathfrak{A})$ admits a $(h, k)$ dichotomy if there exists a sequence of projections $P: \mathbb{N} \rightarrow$ $\mathscr{B}(X)$ and there exist $N, \alpha>0$ and $\beta \geq 0$ such that

$$
\begin{aligned}
& \left(\mathrm{d}_{1}\right) A(m, n) P_{n}=P_{m} A(m, n), \\
& \left(\mathrm{d}_{2}\right)\left\|A(m, n) P_{n} x\right\| \leq N k(n)^{\beta}(h(n) / h(m))^{\alpha}\left\|P_{n} x\right\|, \\
& \left(\mathrm{d}_{3}\right)(h(m) / h(n))^{\alpha}\left\|Q_{n} x\right\| \leq N k(m)^{\beta}\left\|A(m, n) Q_{n} x\right\|,
\end{aligned}
$$

for all $(m, n, x) \in \Delta \times X$.

As we will see below, the $(h, k)$-dichotomy covers a wide range of dichotomy concepts, which are widely used throughout the papers from the bibliography and the references therein (e.g., $[13,14])$. 
Remark 10. As particular cases of $(h, k)$-dichotomy, having in mind the above definition, we have the following:

(i) if $\beta=0$, we have the concept of uniform $(h, k)$ dichotomy;

(ii) if $h(n)=e^{n}$, we have the concept of nonuniform exponential dichotomy;

(iii) if $h(n)=k(n)=e^{n}$, we have the concept of exponential dichotomy;

(iv) if $h(n)=e^{n}$ and $\beta=0$, we have the concept of uniform exponential dichotomy;

(v) if $h(n)=(n+1)$, we have the concept of nonuniform polynomial dichotomy;

(vi) if $h(n)=k(n)=(n+1)$, we have the concept of polynomial dichotomy;

(vii) if $h(n)=(n+1)$ and $\beta=0$, we have the concept of uniform polynomial dichotomy.

Two simple characterizations of the introduced dichotomy concept are given in what follows.

Proposition 11. The linear system ( $\mathfrak{A})$ admits a $(h, k)$ dichotomy if and only if there exists a sequence of projections $P: \mathbb{N} \rightarrow \mathscr{B}(X)$ and there exist $N, \alpha>0$ and $\beta \geq 0$ such that $A(m, n) P_{n}=P_{m} A(m, n)$ for all $(m, n) \in \Delta$ and

$$
\begin{aligned}
& \left\|A(m, n) P_{n} x\right\|+\left\|Q_{n} x\right\| \\
& \quad \leq N\left(\frac{h(n)}{h(m)}\right)^{\alpha}\left[k(n)^{\beta}\left\|P_{n} x\right\|+k(m)^{\beta}\left\|A(m, n) Q_{n} x\right\|\right]
\end{aligned}
$$

for all $(m, n, x) \in \Delta \times X$.

Proof. The necessity follows immediately by adding up $\left(\mathrm{d}_{2}\right)$ and $\left(\mathrm{d}_{3}\right)$. For the sufficiency, let $(m, n) \in \Delta$. For $x \in \operatorname{Range} P_{n}$, we obtain $\left(\mathrm{d}_{2}\right)$, and for $x \in \operatorname{Ker} P_{n}$ we obtain $\left(\mathrm{d}_{3}\right)$.

Proposition 12. The linear system $(\mathfrak{U})$ admits a $(h, k)$ dichotomy if and only if there exists a sequence of projections $P: \mathbb{N} \rightarrow \mathscr{B}(X)$ and there exist $N, \alpha>0$ and $\beta \geq 0$ such that

(i) $\quad A(m, n) P_{n}=P_{m} A(m, n)$;

(ii) $\left\|A\left(m, m_{0}\right) P_{m_{0}} x\right\|$ $\leq N k(n)^{\beta}(h(n) / h(m))^{\alpha}\left\|A\left(n, m_{0}\right) P_{m_{0}} x\right\| ;$

(iii) $\left(\frac{h(m)}{h(n)}\right)^{\alpha}\left\|A\left(n, m_{0}\right) Q_{m_{0}} x\right\|$

$$
\leq N k(m)^{\beta}\left\|A\left(m, m_{0}\right) Q_{m_{0}} x\right\|
$$

for all $(m, n),\left(n, m_{0}\right) \in \Delta$ and for all $x \in X$.
Proof. For the necessity, let $(m, n)$ and $\left(n, m_{0}\right) \in \Delta$ and $x \in X$. We have

$$
\begin{aligned}
& \left\|A\left(m, m_{0}\right) P_{m_{0}} x\right\|=\left\|A(m, n) P_{n} A\left(n, m_{0}\right) P_{m_{0}} x\right\| \\
& \quad \leq N k(n)^{\beta}\left(\frac{h(h)}{h(m)}\right)^{\alpha}\left\|A\left(n, m_{0}\right) P_{m_{0}} x\right\|, \\
& \left(\frac{h(m)}{h(n)}\right)^{\alpha}\left\|A\left(n, m_{0}\right) Q_{m_{0}} x\right\| \\
& \leq N k(m)^{\beta}\left\|A(m, n) Q_{n} A\left(n, m_{0}\right) Q_{m_{0}} x\right\| \\
& =N k(m)^{\beta}\left\|A\left(m, m_{0}\right) Q_{m_{0}} x\right\| .
\end{aligned}
$$

The sufficiency follows from choosing $n=m_{0}$.

In what follows we will present the main results of our paper.

Theorem 13. Let $(\mathfrak{A})$ be a linear system such that there exist two growth rates $h, k: \mathbb{N} \rightarrow[1,+\infty)$ with the following properties:

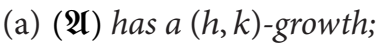

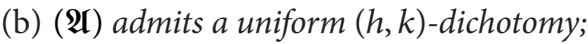

(c) there exist $G, H: \mathbb{N} \rightarrow(0,+\infty)$ with $\lim _{n \rightarrow+\infty} G(n)=$ $+\infty$ such that

$$
G(m-n) \leq \frac{h(m)}{h(n)} \leq H(m-n)
$$

for all $(m, n) \in \Delta$.

If $P: \mathbb{N} \rightarrow \mathscr{B}(X)$ is a family of projections that gives the $(h, k)$-dichotomy of the system $(\mathfrak{U})$, then $P$ is $k$-bounded.

Proof. Let $N, \alpha$ and $P: \mathbb{N} \rightarrow \mathscr{B}(X)$ be given from the uniform $(h, k)$-dichotomy property and $M, \omega_{1}, \omega_{2}$ the constants from the $(h, k)$-growth property of $(\mathfrak{H})$. Let $n \in \mathbb{N}$ and $x \in X$ be fixed and consider $m \geq n$. From the fact that

$$
\begin{aligned}
& \frac{1}{N}\left(\frac{h(m)}{h(n)}\right)^{\alpha}\left(\left\|P_{n} x\right\|-\|x\|\right)-N\left(\frac{h(n)}{h(m)}\right)^{\alpha}\left\|P_{n} x\right\| \\
& \quad \leq \frac{1}{N}\left(\frac{h(m)}{h(n)}\right)^{\alpha}\left\|Q_{n} x\right\|-N\left(\frac{h(n)}{h(m)}\right)^{\alpha}\left\|P_{n} x\right\| \\
& \quad \leq\left\|A(m, n) Q_{n} x\right\|-\left\|A(m, n) P_{n} x\right\| \\
& \quad \leq\|A(m, n) x\| \\
& \quad \leq M k(n)^{\omega_{2}}\left(\frac{h(m)}{h(n)}\right)^{\omega_{1}}\|x\|
\end{aligned}
$$

by using (c), we obtain that

$$
\begin{aligned}
& {\left[\frac{1}{N} G(m-n)^{\alpha}-\frac{N}{H(m-n)^{\alpha}}\right]\left\|P_{n} x\right\|} \\
& \quad \leq M k(n)^{\omega_{2}} H(m-n)^{\max \left\{\alpha, \omega_{1}\right\}}\|x\| .
\end{aligned}
$$


By the hypothesis, $G(j) \leq H(j)$, for all $j \in \mathbb{N}$. Because $\lim _{j \rightarrow+\infty} G(j)=+\infty$, it follows that $\lim _{j \rightarrow+\infty} H(j)=+\infty$ and therefore

$$
\lim _{j \rightarrow+\infty}\left[\frac{1}{N} G(j)^{\alpha}-\frac{N}{H(j)^{\alpha}}\right]=+\infty ;
$$

hence there exists $m_{0} \in \mathbb{N}$ such that

$$
L:=\frac{M H\left(m_{0}\right)^{\max \left\{\alpha, \omega_{1}\right\}}}{(1 / N) G\left(m_{0}\right)^{\alpha}-\left(N / H\left(m_{0}\right)^{\alpha}\right)}>0 .
$$

By choosing $m=n+m_{0}$, taking into account (16) we finally have that

$$
\left\|P_{n} x\right\| \leq L k(n)^{\omega_{2}}\|x\| \quad \forall x \in X, \forall n \in \mathbb{N} .
$$

Corollary 14. Let $(\mathfrak{U})$ be a linear system such that there exist two growth rates $h, k: \mathbb{N} \rightarrow[1,+\infty)$ with the following properties:

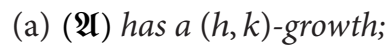

(b) ( $\mathfrak{A})$ admits a uniform $(h, k)$-dichotomy;

(c) there exist $H: \mathbb{N} \rightarrow(0,+\infty)$ such that

$$
\frac{h(m)}{h(n)}=H(m-n)
$$

for all $(m, n) \in \Delta$.

If $P: \mathbb{N} \rightarrow \mathscr{B}(X)$ is a family of projections that gives the $(h, k)$-dichotomy of the system $(\mathfrak{A})$, then $P$ is $k$-bounded.

Remark 15. In contrast with the condition (c) from Theorem 13, the condition (c) from Corollary 14 is rather restrictive; namely, such a pair $(h, H)$ is quite particular. Indeed, from (c), having in mind that $h$ is a growth rate, it follows that, for all $n \in \mathbb{N}$,

$$
\frac{h(m)}{h(0)}=h(m)=H(m)
$$

hence

$$
\frac{h(m)}{h(n)}=h(m-n), \quad \forall(m, n) \in \Delta .
$$

Let $n \in \mathbb{N}$ and $m=n+1$. It follows that $h(n+1) / h(n)=h(1)$ from where

$$
h(n)=h(1)^{n}, \quad \forall n \in \mathbb{N} .
$$

By setting $\gamma=\ln h(1) \geq 0$ we obtain that

$$
H(n)=h(n)=e^{\gamma n}, \quad \forall n \in \mathbb{N}
$$

showing us that the behavior of the system $(\mathfrak{H})$ is exponential.

Corollary 16. If the system ( $\mathfrak{U})$ has exponential growth and it is uniformly exponentially dichotomic, then there exists a sequence of projections that gives the specified dichotomic behavior of the system $(\mathfrak{A})$ and it is also exponentially bounded.
Theorem 17. Let $(\mathfrak{A})$ be a linear system such that there exist two growth rates $h, k: \mathbb{N} \rightarrow[1,+\infty)$ with the following properties:

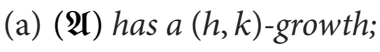

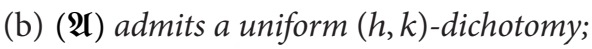

(c) $\mathbb{N}^{*} \subset$ Range $(h)$.

If $P: \mathbb{N} \rightarrow \mathscr{B}(X)$ is a family of projections that gives the $(h, k)$-dichotomy of the system $(\mathfrak{A})$, then $P$ is $k$-bounded.

Proof. Let $N, \alpha$ and $P: \mathbb{N} \rightarrow \mathscr{B}(X)$ be given from the uniform $(h, k)$-dichotomy property and the constants $M, \omega_{1}, \omega_{2}$ form the $(h, k)$-growth property of $(\boldsymbol{\Re})$. Let $n \in \mathbb{N}$ and $x \in X$ be fixed. As in the proof from Theorem 13 we obtain the inequality

$$
\begin{aligned}
& {\left[\frac{1}{N}\left(\frac{h(m)}{h(n)}\right)^{\alpha}-N\left(\frac{h(n)}{h(m)}\right)^{\alpha}\right]\left\|P_{n} x\right\|} \\
& \quad \leq M k(n)^{\omega_{2}}\left(\frac{h(m)}{h(n)}\right)^{\max \left\{\alpha, \omega_{1}\right\}}\|x\|
\end{aligned}
$$

valid for all $m \geq n$.

Let $n_{0} \in \mathbb{N}, n_{0} \geq 2$ be such that

$$
L_{0}:=\frac{M N n_{0}^{\alpha+\max \left\{\alpha, \omega_{1}\right\}}}{n_{0}^{2 \alpha}-N^{2}}>0 .
$$

By (c) we may choose $m \in \mathbb{N}$ such that

$$
h(m)=n_{0} h(n) .
$$

It is easy to see that $(m, n) \in \Delta$ because if we assume the contrary, from $m<n$, using $\left(\mathrm{gr}_{1}\right)$, we get $h(m) \leq h(n)$ and $n_{0} \cdot h(n) \leq h(n)$ which, from the fact that $h(n) \neq 0$, gives us $n_{0} \leq 1$, which contradicts the choice of $n_{0}$.

From (25), (26), and (27), it follows that

$$
\left\|P_{n} x\right\| \leq L_{0} k(n)^{\omega_{2}}\|x\|
$$

for all $n \in \mathbb{N}$ and for all $x \in X$; hence $P$ is $k$-bounded.

Corollary 18. If the system ( $\mathfrak{U})$ has polynomial growth and it is uniformly polynomially dichotomic, then there exists a sequence of projections that gives the assumed polynomial behavior of the system $(\mathfrak{A})$ which is polynomially bounded.

Indeed, if $k(n)=h(n)=n+1$, it is easy to see that

$$
\text { Range }(h)=\mathbb{N}^{*}
$$

and by applying the preceding result, we obtain the desired conclusion.

Remark 19. If, for the system $(\mathfrak{A})$, the $(h, k)$-growth is uniform and the $(h, k)$-dichotomy is as well uniform, then the given sequence of projections that gives the dichotomy property is bounded. 
Remark 20. In order to prove the $k$-boundedness of the sequence of projections in the case of uniform asymptotic behavior, the $(h, k)$-growth is essential, as we will see in the following example. Moreover, by the following choice of the system to depend on the sequence of projections, we also intend to point out how the growth and the dichotomy are strongly connected to each other, through the same growth rate $k$.

We will give a particular example, in the exponential framework.

Example 21. Consider the growth rates $h, k: \mathbb{R}_{+} \rightarrow[1, \infty)$ defined by

$$
h(n)=e^{n}, \quad k(n)=(n+1)^{2} \quad n \in \mathbb{N} .
$$

On $X=\mathbb{R}^{2}$ endowed with the norm

$$
\|x\|=\max \left\{\left|x_{1}\right|,\left|x_{2}\right|\right\} \quad \text { for } x=\left(x_{1}, x_{2}\right) \in \mathbb{R}^{2}
$$

define the sequence of projections $P: \mathbb{N} \rightarrow \mathscr{B}\left(\mathbb{R}^{2}\right)$ by

$$
P_{n} x=\left(x_{1}+e^{k(n)} x_{2}, 0\right) \quad \forall(n, x) \in \mathbb{N} \times \mathbb{R}^{2} .
$$

Let $m, n \in \mathbb{N}$ and $x=\left(x_{1}, x_{2}\right) \in \mathbb{R}^{2}$. We observe that

$$
\begin{aligned}
P_{m} P_{n} x & =P_{m}\left(x_{1}+e^{k(n)} x_{2}, 0\right) \\
& =\left(x_{1}+e^{k(n)} x_{2}+e^{k(m)} \cdot 0,0\right)=P_{m} x ;
\end{aligned}
$$

hence

$$
P_{m} P_{n}=P_{n} \quad \forall m, n \in \mathbb{N} \text {. }
$$

Moreover, if $Q: \mathbb{N} \rightarrow \mathscr{B}\left(\mathbb{R}^{2}\right)$ is the complementary sequence of $P$, defined by

$$
\begin{gathered}
Q_{n} x=\left(I-P_{n}\right) x=\left(-e^{(n+1)^{2}} x_{2}, x_{2}\right) \\
\forall n \in \mathbb{N}, \quad x=\left(x_{1}, x_{2}\right) \in \mathbb{R}^{2},
\end{gathered}
$$

we have that for all $m, n \in \mathbb{N}$

$$
\begin{aligned}
Q_{m} Q_{n} & =\left(I-P_{m}\right)\left(I-P_{n}\right)=I-P_{m}-P_{n}+P_{m} P_{n} \\
& =I-P_{m}-P_{n}+P_{n}=I-P_{m}=Q_{m} ;
\end{aligned}
$$

hence

$$
Q_{m} Q_{n}=Q_{m} \quad \forall m, n \in \mathbb{N} .
$$

As a consequence of the above, we also have that for all $m, n \in$ $\mathbb{N}$

$$
Q_{m} P_{n}=Q_{m} P_{m} P_{n}=0 .
$$

In addition, another property that we will use in what follows is given by the fact that if $(m, n) \in \Delta$ and $x=\left(x_{1}, x_{2}\right) \in \mathbb{R}^{2}$, we have that

$$
\begin{aligned}
\left\|Q_{m} x\right\| & =\left\|\left(-e^{k(m)} x_{2}, x_{2}\right)\right\| \\
& =\max \left\{e^{k(m)}\left|x_{2}\right|,\left|x_{2}\right|\right\}=e^{k(m)}\left|x_{2}\right| \geq e^{k(n)}\left|x_{2}\right| \\
& =\left\|Q_{n} x\right\| .
\end{aligned}
$$

Consider the sequence of operators $\mathbb{A}: \mathbb{N} \rightarrow \mathscr{B}(X)$ given by

$$
\mathbb{A}_{n}=\frac{1}{e} P_{n}+e Q_{n+1} \quad \forall n \in \mathbb{N}
$$

and the corresponding linear system $(\mathfrak{A})$.

Next we will give the expression of the evolution operator which governs $(\mathfrak{\mathcal { U }})$.

For $(m, n) \in \Delta$ with $m \neq n$, one can see that

$$
\begin{aligned}
& \mathbb{A}_{m-1} \mathbb{A}_{m-2} \cdots \mathbb{A}_{n} \\
&=\left(\frac{1}{e} P_{m-1}+e Q_{m}\right)\left(\frac{1}{e} P_{m-2}+e Q_{m-1}\right) \\
& \cdots\left(\frac{1}{e} P_{n}+e Q_{n+1}\right) \\
&=\left(\frac{1}{e^{2}} P_{m-1} P_{m-2}+P_{m-1} Q_{m-1}+Q_{m} P_{m-2}+e^{2} Q_{m} Q_{m-1}\right) \\
& \cdots\left(\frac{1}{e} P_{n}+e Q_{n+1}\right) \\
&=\left(\frac{1}{e^{2}} P_{m-2}+e^{2} Q_{m}\right) \cdots\left(\frac{1}{e} P_{n}+e Q_{n+1}\right) \\
&= \cdots=e^{-(m-n)} P_{n}+e^{m-n} Q_{m} .
\end{aligned}
$$

Thus we have

$$
A(m, n)=e^{-(m-n)} P_{n}+e^{m-n} Q_{m}, \quad \forall(m, n) \in \Delta .
$$

Consider now $(m, n) \in \Delta$ and $x=\left(x_{1}, x_{2}\right) \in \mathbb{R}^{2}$. We will prove that $(\mathfrak{A})$ is uniformly $(h, k)$-dichotomic with $h(n)=e^{n}$, $k(n)=(n+1)^{2}, N=\alpha=1$, and $\beta=0$. Consider the following:

$$
\begin{aligned}
\left\|A(m, n) P_{n} x\right\| & =\left\|\left(e^{-(m-n)} P_{n}+e^{m-n} Q_{m}\right) P_{n} x\right\| \\
& =\left\|e^{-(m-n)} P_{n} x+e^{m-n} Q_{m} P_{n} x\right\| \\
& =e^{-(m-n)}\left\|P_{n} x\right\|, \\
\left\|A(m, n) Q_{n} x\right\| & =\left\|\left(e^{-(m-n)} P_{n}+e^{m-n} Q_{m}\right) Q_{n} x\right\| \\
& =\left\|e^{-(m-n)} P_{n} Q_{n} x+e^{m-n} Q_{m} Q_{n} x\right\| \\
& =e^{m-n}\left\|Q_{m} x\right\| \geq e^{m-n}\left\|Q_{n} x\right\|
\end{aligned}
$$

from where the conclusion follows.

The $k$-boundedness of the sequence of projections $P$ fails to hold, mainly because the $(h, k)$-growth of the system $(\mathfrak{\mathcal { I }})$ does not hold.

Indeed, on one hand we have that

$$
\left\|P_{n}\right\|=e^{k(n)}+1, \quad \forall n \in \mathbb{N}
$$

from where the $k$-boundedness fails.

On the other hand, assume that there exist $M \geq 1, \omega_{1}>0$, and $\omega_{2} \geq 0$ such that the system $(\mathfrak{U})$ has a $(h, k)$-growth. Let $n \in \mathbb{N}$ and $x_{n}=\left(-e^{k(n)}, 1\right) \in \mathbb{R}^{2}$ with

$$
\left\|x_{n}\right\|=e^{k(n)}, \quad P_{n} x_{n}=0, \quad Q_{n+1} x_{n}=\left(-e^{k(n+1)}, 1\right) .
$$


Then, by (10), we have in particular that

$$
\left\|A(n+1, n) x_{n}\right\| \leq M e^{\omega_{1}}(n+1)^{2 \omega_{2}}\left\|x_{n}\right\| .
$$

Having in mind that

$$
\left\|A(n+1, n) x_{n}\right\|=\left\|\frac{1}{e} P_{n} x_{n}+e Q_{n+1} x_{n}\right\|=e \cdot e^{(n+2)^{2}}
$$

by (47) it follows that

$$
e \cdot e^{(n+2)^{2}} \leq M e^{\omega_{1}}(n+1)^{2 \omega_{2}} \cdot e^{(n+1)^{2}}
$$

which leads us to the contradicting inequality

$$
\frac{e^{2 n+3}}{(n+1)^{2 \omega_{2}}} \leq M e^{\omega_{1}-1}, \quad \forall n \in \mathbb{N} .
$$

It follows that the system $(\mathfrak{P})$ does not admit a $(h, k)$ growth.

Remark 22. In the preceding example, if we consider the family of projections

$$
\begin{array}{r}
\widetilde{P}: \mathbb{N} \longrightarrow \mathscr{B}\left(\mathbb{R}^{2}\right), \quad \widetilde{P}_{n} x=\left(x_{1}+(k(n)-1) x_{2}, 0\right), \\
\text { for }(n, x) \in \mathbb{N} \times \mathbb{R}^{2}
\end{array}
$$

and denote $\widetilde{Q}_{n}=I-\widetilde{P}_{n}$, then the associated discrete system has the evolution operator $\widetilde{A}: \Delta \rightarrow \mathscr{B}\left(\mathbb{R}^{2}\right)$ defined by

$$
\widetilde{A}(m, n)=e^{-(m-n)} \widetilde{P}_{n}+e^{m-n} \widetilde{Q}_{m}, \quad(m, n) \in \Delta .
$$

By similar arguments as in the previous example, we have that the system described by $\widetilde{A}: \Delta \rightarrow \mathscr{B}\left(\mathbb{R}^{2}\right)$ is also uniformly $(h, k)$-dichotomic and

$$
\left\|\widetilde{P}_{n}\right\|=k(n) \quad \forall n \in \mathbb{N}
$$

hence $P$ is $k$-bounded. Thus, along with the counterexample presented from above with $P$ not being $k$-bounded, there are systems which verify the same property and the sequence of projections is $k$-bounded. More examples of discrete systems and $k$-(un)bounded sequences of projections can be found in [13].

\section{Conflict of Interests}

The authors declare that there is no conflict of interests regarding the publication of this paper.

\section{Acknowledgments}

Mihai-Gabriel Babuţia was supported by a Grant of the Romanian National Authority for Scientific Research, CNCS UEFISCDI, Project no. PN-II-RU-TE-2011-3-0103. The authors would like to thank the referees for their suggestions that led to the final form of the paper.

\section{References}

[1] A. I. Alonso, J. Hong, and R. Obaya, "Exponential dichotomy and trichotomy for difference equations," Computers and Mathematics with Applications, vol. 38, no. 1, pp. 41-49, 1999.

[2] L. Barreira and C. Valls, "Robustness of a weaker notion of exponential dichotomy," Integral Equations and Operator Theory, vol. 79, no. 3, pp. 337-354, 2014.

[3] S. Elaydi and K. Hanglajew, "Dichotomy and trichotomy of difference equations," Journal of Difference Equations and Applications, vol. 3, no. 5-6, pp. 417-448, 1998.

[4] M. Megan, A. L. Sasu, and B. Sasu, "Discrete admissibility and exponential dichotomy for evolution families," Discrete and Continuous Dynamical Systems, vol. 9, no. 2, pp. 383-397, 2003.

[5] M. Pinto, "Discrete dichotomies," Computers and Mathematics with Applications, vol. 28, pp. 259-270, 1994.

[6] I. Popa, M. Megan, and T. Ceausu, "Exponential dichotomies for linear discrete-time systems in Banach spaces," Applicable Analysis and Discrete Mathematics, vol. 6, no. 1, pp. 140-155, 2012.

[7] I. L. Popa, M. Megan, and T. Ceausu, "Nonuniform exponential dichotomies in terms of Lyapunov functions for noninvertible linear discrete-time systems," The Scientific World Journal, vol. 2013, Article ID 901026, 7 pages, 2013.

[8] A. L. Sasu and B. Sasu, "Discrete admissibility and exponential trichotomy of dynamical systems," Discrete and Continuous Dynamical Systems, vol. 34, no. 7, pp. 2929-2962, 2014.

[9] A. L. Sasu and B. Sasu, "Exponential trichotomy for variational difference equations," Journal of Difference Equations and Applications, vol. 15, no. 7, pp. 693-718, 2009.

[10] B. Sasu, "On exponential dichotomy of variational difference equations," Discrete Dynamics in Nature and Society, vol. 2009, Article ID 324273, 18 pages, 2009.

[11] B. Sasu and A. L. Sasu, "Exponential dichotomy and $l^{p}, l^{q}$ admissibility on the half-line," Journal of Mathematical Analysis and Applications, vol. 316, no. 2, pp. 397-408, 2006.

[12] B. Sasu and A. L. Sasu, "On the dichotomic behavior of discrete dynamical systems on the half-line," Discrete and Continuous Dynamical Systems. Series A, vol. 33, no. 7, pp. 3057-3084, 2013.

[13] M. G. Babutia, M. Megan, and I. L. Popa, "On $(h, k)$ dichotomies for nonautonomous difference equations in Banach spaces," International Journal of Differential Equations, vol. 2013, Article ID 761680, 7 pages, 2013.

[14] A. J. G. Bento and C. M. Silva, "Nonuniform $(\mu, \nu)$-dichotomies and local dynamics of difference equations," Nonlinear Analysis: Theory, Methods and Applications, vol. 75, no. 1, pp. 78-90, 2012.

[15] J. López-Fenner and M. Pinto, “ $(h, k)$-trichotomies and asymptotics of nonautonomous difference systems," Computers and Mathematics with Applications, vol. 33, no. 10, pp. 105-124, 1997.

[16] M. Megan and L. Buliga, "Nonuniform exponential trichotomy for difference equations in Banach spaces," in Hot Topics in Operator Theory, vol. 9 of Theta Series in Advanced Mathematics, pp. 145-150, Theta, 2008.

[17] G. Papaschinopoulos, "On exponential trichotomy of linear difference equations," Journal of Applied Analysis, vol. 40, pp. 89-109, 1991. 


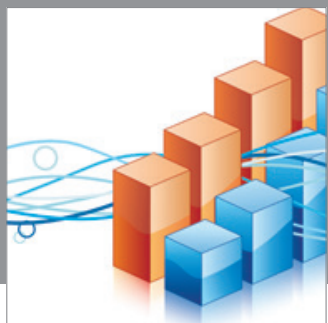

Advances in

Operations Research

mansans

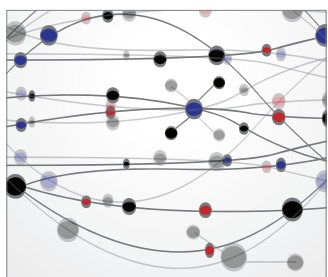

The Scientific World Journal
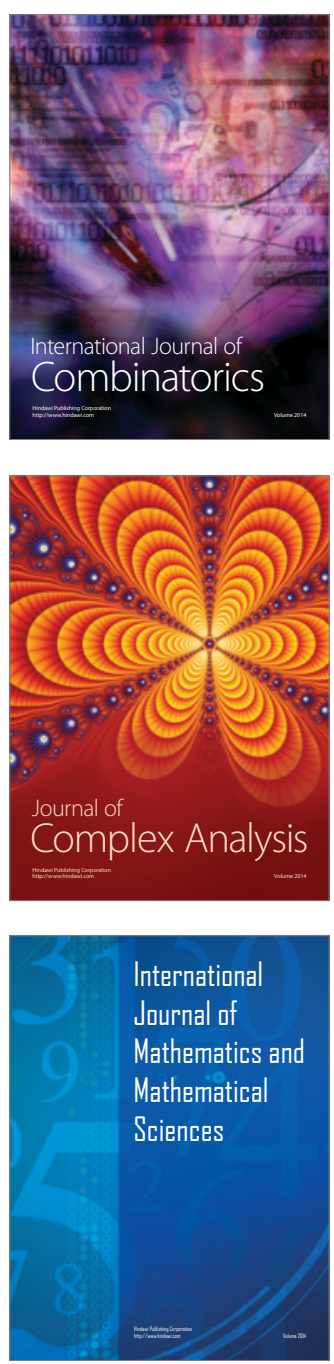
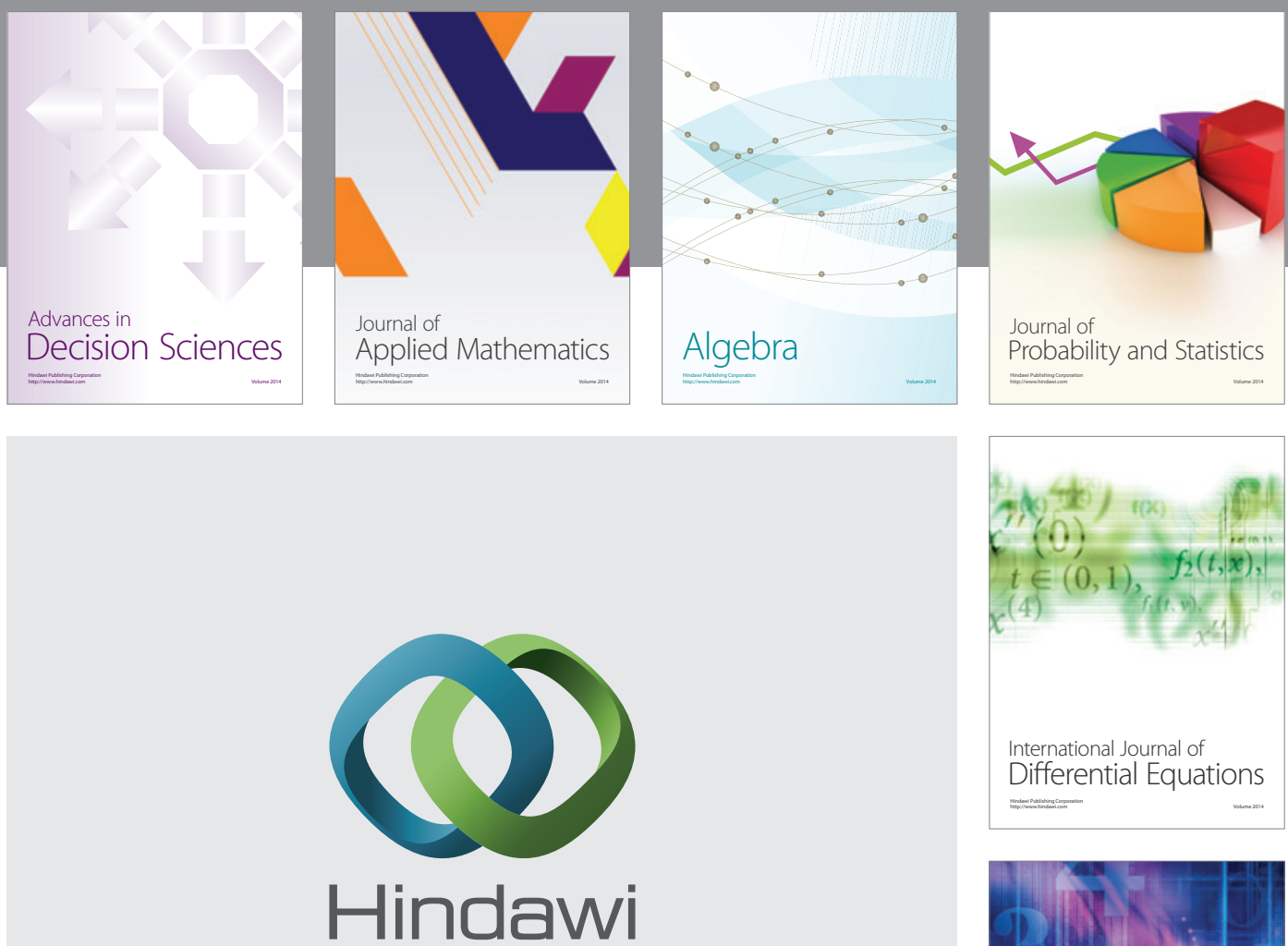

Submit your manuscripts at http://www.hindawi.com
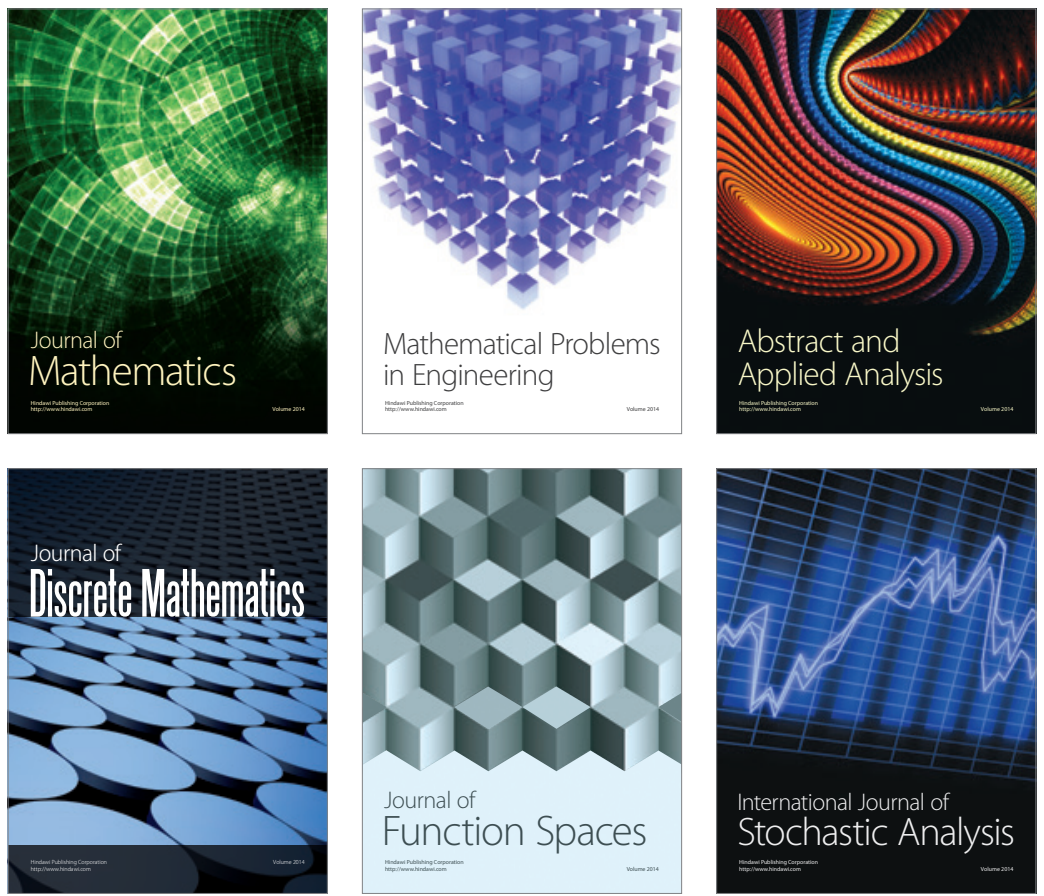

Journal of

Function Spaces

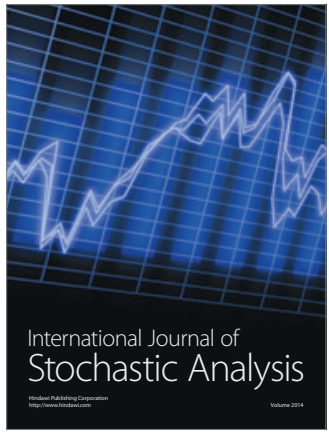

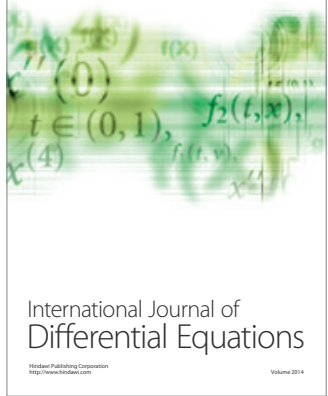
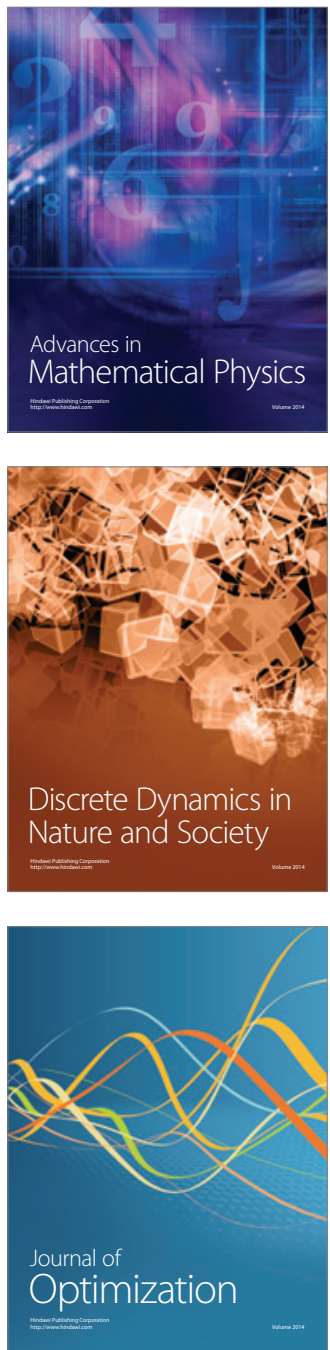